\title{
Biological therapy for the treatment of prepouch ileitis: a retrospective observational study from three centers
}

This article was published in the following Dove Press journal:

Clinical and Experimental Gastroenterology

\author{
Jonathan P Segal ${ }^{1,2}$ \\ Matteo Rottoli ${ }^{3}$ \\ Richard K Felwick ${ }^{4}$ \\ Guy HT Worley ${ }^{1,2}$ \\ Simon D McLaughlin ${ }^{4}$ \\ Carlo Vallicelli ${ }^{3}$ \\ Paul Bassett ${ }^{5}$ \\ Omar D Faiz',2 \\ Ailsa L Hart ${ }^{1,2}$ \\ Susan K Clark ${ }^{1,2}$ \\ 'Inflammatory Bowel Disease \\ Department, St Mark's Hospital, \\ Harrow, UK; ' ${ }^{2}$ Department of Surgery \\ and Cancer, Imperial College, London, \\ UK; ${ }^{3}$ Surgery of the Alimentary Tract, \\ Sant'Orsola - Malpighi Hospital, \\ Alma Mater Studiorum University of \\ Bologna, Bologna, Italy; ${ }^{4}$ Department \\ of Gastroenterology, The Royal \\ Bournemouth and Christchurch \\ Hospitals, Bournemouth, UK; \\ ${ }^{5}$ Statsconsultancy Ltd, Amersham, UK
}

Correspondence: Jonathan P Segal Inflammatory Bowel Disease Department, St Mark's Hospital, Watford Road,

Harrow, HAI 3UJ, UK

Tel +447809148950

Email Jonathansegal@doctors.org.uk
Aim: Prepouch ileitis (PPI) is inflammation of the ileum proximal to an ileoanal pouch, usually associated with pouchitis. The treatment of PPI as a specific entity has been poorly studied, but it is generally treated concurrently with pouchitis. This to our knowledge is the largest study to explore the efficacy of biologics for the specific treatment of PPI.

Methods: This was a retrospective observational study reporting outcomes following biological treatment in patients with PPI across three centers. Data were collected between January 2004 and February 2018 from two centers in the UK and one center in Italy. Outcomes included the continued presence of PPI following biologic therapy, pouch failure defined by the need for an ileostomy, and remission of PPI defined by the absence of any prepouch inflammation on endoscopic assessment within a year of biologic therapy.

Results: There were 29 patients in our cohort. On last endoscopic follow-up, 20/29 still had endoscopic evidence of PPI, seven had achieved endoscopic remission and avoided an ileostomy, and two had no endoscopic follow-up. In our cohort 11 patients had an ileostomy after a median time from starting a biologic of 25 months (range 14-91).

Conclusion: Biologics fail to induce endoscopic remission of PPI in the majority of patients. Just under one-third patients with PPI coexistent with pouchitis can achieve endoscopic remission with biologics. In a large proportion of patients with PPI, surgery may be required despite biologic use.

Keywords: pouch, biologics, prepouch ileitis, pouchitis, restorative proctocolectomy, infliximab

\section{Introduction}

\section{Background and rationale}

Restorative proctocolectomy (RPC) with ileal pouch-anal anastomosis is considered the procedure of choice in patients with ulcerative colitis (UC) refractory to medical therapy and in those who develop colonic dysplasia or cancer. ${ }^{1}$ The procedure is performed with the aim of improving quality of life with generally good outcomes; ${ }^{2,3}$ however, complications including pouchitis can arise. The incidence of acute pouchitis is $20 \%$ at 1 year and up to $40 \%$ at 5 years following surgery. ${ }^{4}$ Chronic idiopathic pouchitis develops in $\sim 10 \%-15 \%$ of patients with acute pouchitis. ${ }^{5,6}$ Another less well-reported complication is prepouch ileitis (PPI).

PPI has no standard definition but is inflammation of the ileum proximal to the pouch. The estimated frequency of PPI is $6 \% .^{7,8}$ This pattern of inflammation can extend for a significant distance into the afferent limb (up to $50 \mathrm{~cm}$ ), ${ }^{9}$ but this is unusual. ${ }^{8}$ It is usually seen with coexisting pouchitis. It is mostly a condition that is associated 
with a pouch for UC and is rarely seen in patients who have undergone a pouch for familial adenomatous polyposis. ${ }^{9}$ This therefore suggests that both the immune system and the environment interact within a genetically susceptible patient to cause inflammation.

As the immune system is likely to play a role in PPI, the use of biologic medications may potentially help achieve remission through the immunomodulatory actions. Furthermore, biologic drugs have been shown to result in remission in about $50 \%$ of patients with inflammation of their pouch. ${ }^{10}$ As it has been shown that PPI is often associated with pouchitis, it may therefore be beneficial in treating this problem. ${ }^{8,11}$

Symptoms of PPI are generally nonspecific but can include increased stool frequency, outlet obstruction, and bleeding. ${ }^{12}$ The treatment of PPI as a specific entity has been poorly studied, but it is generally treated concurrently with pouchitis. One small study looked at the effects of antibiotics on PPI in a cohort with a diagnosis of UC and showed that $86 \%$ of patients showed symptomatic improvement with significant reduction in both stool frequency and length of PPI. However, this was based on only 14 patients. Furthermore, there has been only one small case series of five patients where infliximab was found to be effective in the short term for the specific treatment of PPI in a cohort with a diagnosis of Crohn's disease. ${ }^{13}$

Historically, the limited literature reporting outcomes of biologic therapy for PPI has assumed that PPI is a Crohn'slike complication. ${ }^{13-15}$ Recent studies, however, have highlighted that PPI is not a strong predictor for the development of unequivocal features of Crohn's disease, ${ }^{11,12}$ and therefore, it is important to report treatment outcomes dependent on whether Crohn's disease is thought to be the underlying cause or not.

\section{Objectives}

This aim of this study was to document the efficacy of biologics for the treatment of PPI. To our knowledge, this is the largest study to explore the efficacy of all biologics for the specific treatment of PPI with the longest follow-up data.

\section{Methods}

\section{Study design and setting}

This was a retrospective observational study across three centers. Data were collected from two centers in the UK and one center in Bologna, Italy. This included two tertiary referral centers and one district general hospital. Patients were censored at the last clinical encounter following their most recent biologic therapy or until they had pouch failure defined by the need to form an ileostomy to relieve symptoms.

\section{Participants}

Patients were included if they met all of the following inclusion criteria:

- Undergone restorative proctocolectomy for UC

- Evidence of PPI on endoscopic assessment with inflammation confirmed histologically

- Treated with infliximab, adalimumab, or vedolizumab (other biologics were not available at our centers during the study period)

\section{Variables}

Patients were followed up until last clinical encounter. Outcomes included the presence of PPI following biologic therapy, pouch failure defined by the need for an ileostomy, remission of PPI defined by the absence of any prepouch inflammation on endoscopic assessment within a year of biologic therapy and the need to switch to an alternative biologic. Primary nonresponse was defined as failure of clinical improvement at 12 weeks of biologic therapy. Secondary loss of response was defined as lack of clinical response to biologic therapy after 12 weeks of treatment.

Lack of clinical benefit was judged by the senior clinician looking after the patient and was guided by symptoms, endoscopic, histological, and biochemical markers.

A template data collection sheet was given to each subinvestigator to collect the variables of interest. Potential patients were identified using each hospital's biologics and pouch databases. Those patients identified from the database were then screened against the inclusion and exclusion criteria by interrogation of the patient's electronic and case notes. Completed templates were then collated and analyzed.

\section{Measurement of variables}

The use of the term Crohn's disease (CD) is controversial in pouch-related inflammatory problems. ${ }^{16}$ In our study, we defined this by the presence of conclusive histology (granulomas supporting CD) and/or presence of skip lesions in the small bowel. Pouchitis was defined using the pouch disease activity index (PDAI). ${ }^{17}$ Patients were classified as having pouchitis if their PDAI within 1 year before starting infliximab was $\geq 7$. PPI was defined as any inflammation immediately proximal to the pouch; inflammation was defined if the endoscopist reported the presence of edema, 
ulceration, erythema, or contact bleeding in the immediate prepouch ileum with histological confirmation of inflammation in that section.

\section{Statistical methods}

All variables were analyzed using STATA (StataCorp LP, College Station, TX, USA).

\section{Ethical approval}

Ethical approval was granted by the Health Research Authority (IRAS ID: 233311).

The study was considered a service evaluation study, and hence as anonymized data were used, written consent was not required by our ethics committee. Local ethics was not considered a requirement by Malpighi Hospital as the study was considered an audit of practice. The study was carried out in accordance with the principles of the declaration of Helsinki.

\section{Results}

There were 29 patients in our cohort. The median age of diagnosis of UC was 27 years (range 6-48 years). The median age of the patients at censorship was 53 years (range 19-68 years). The median time from pouch formation to diagnosis of PPI was 79 months (range 1-147 months). The median length of time a patient was on biologics at censorship was 12 months (range 2-62 months). The median length of follow-up on the whole cohort was 21 months (range 1-99 months) (Table 1).

Ten patients were reclassified as having confirmed CD after a median time from formation of pouch of 202 months (range 1-372 months; Figure 1). Six had granulomas on

Table I Baseline characteristics

\begin{tabular}{|l|l|l|}
\hline Variable & Category & N (\%) \\
\hline Total patients & & $29(100)$ \\
\hline \multirow{2}{*}{ Gender } & Male & I7 (58) \\
\cline { 2 - 3 } & Female & $12(4 \mathrm{I})$ \\
\hline \multirow{2}{*}{$\begin{array}{l}\text { diagn subtype at } \\
\text { censorship }\end{array}$} & UC & $28(96)$ \\
\cline { 2 - 3 } $\begin{array}{l}\text { Primary sclerosing } \\
\text { cholangitis }\end{array}$ & Indeterminate colitis & $\mathrm{I}(4)$ \\
\hline \multirow{2}{*}{$\begin{array}{l}\text { Initial indication for } \\
\text { biologic }\end{array}$} & Yes & $9(3 \mathrm{I})$ \\
\cline { 2 - 3 } & No & $20(69)$ \\
\hline & Yes & $3(10)$ \\
\hline Initial biologic used & No & $26(90)$ \\
\cline { 2 - 3 } & PPI in isolation & $22(76)$ \\
\cline { 2 - 3 } & CD with PPI & $3(10)$ \\
\hline $\begin{array}{l}\text { Prior exposure to } \\
\text { biologics before pouch }\end{array}$ & Infliximab & $4(14)$ \\
\cline { 2 - 3 } & Adalimumab & $20(69)$ \\
\hline
\end{tabular}

Abbreviations: $C D$, Crohn's disease; IBD, inflammatory bowel disease; PPI, prepouch ileitis; UC, ulcerative colitis. further histological assessment and skip lesions on small bowel imaging and four had granulomas on histology alone.

\section{Change of medications}

One patient had primary nonresponse to infliximab and was changed to vedolizumab. Nine other patients had secondary loss of response to infliximab; of these, six were changed to adalimumab and three were changed to vedolizumab. Of all those in whom the first biologic failed, the median time to failure was 12.0 months (range 2-39 months).

\section{Remission and pouch failure}

At last endoscopic follow-up within 1 year of starting a biologic, 20 of 29 patients still had endoscopic evidence of PPI, seven had achieved remission, and two had no endoscopic follow-up. Of the six patients who achieved endoscopic remission, four had a biopsy from the prepouch ileum which demonstrated histological remission. The other two were not biopsied at endoscopic follow-up. Of the seven who had achieved remission, five have stopped all medications and remained clinically well, one was taking colifoam enemas and one used cyclical metronidazole to maintain symptomatic response. All patients who had achieved remission were patients who had their biologic for PPI with coexisting pouchitis.

In our cohort, 11 patients went on to pouch failure after a median time from starting a biologic of 25 months (range 14-91 months). Of those who had their UC reclassified to $\mathrm{CD}$, three of ten patients $(30 \%)$ had pouch failure compared with eight of $19(42 \%)$ who had UC $(P=0.72)$ (Figure 1). The cumulative 1-, 2-, 5-, and 8 -year failure rates were $0 \%, 17 \%$, $30 \%$, and $38 \%$, respectively (Figure 1).

\section{Discussion}

PPI remains a difficult condition to treat. Our study has highlighted that the use of biologics for PPI is associated with relatively low remission rates of $31 \%$ at a median follow-up of 20.5 months (range 1-99 months). When using biologics for PPI, there was pouch failure in just over one third of the cohort after a median follow-up of 25 months (range 14-91 months). In our small cohort, we found that PPI associated with CD had lower rates of failure than PPI with UC but this was not statistically significant. Although it is hard to draw conclusions in a small study, it is possible that PPI associated with CD has different response rates to PPI associated with UC but this will require further exploration in larger prospective studies.

There are a paucity of data highlighting the outcomes of biologic treatments in inflammatory pouch problems. A systematic review that included all chronic inflammatory pouch problems 


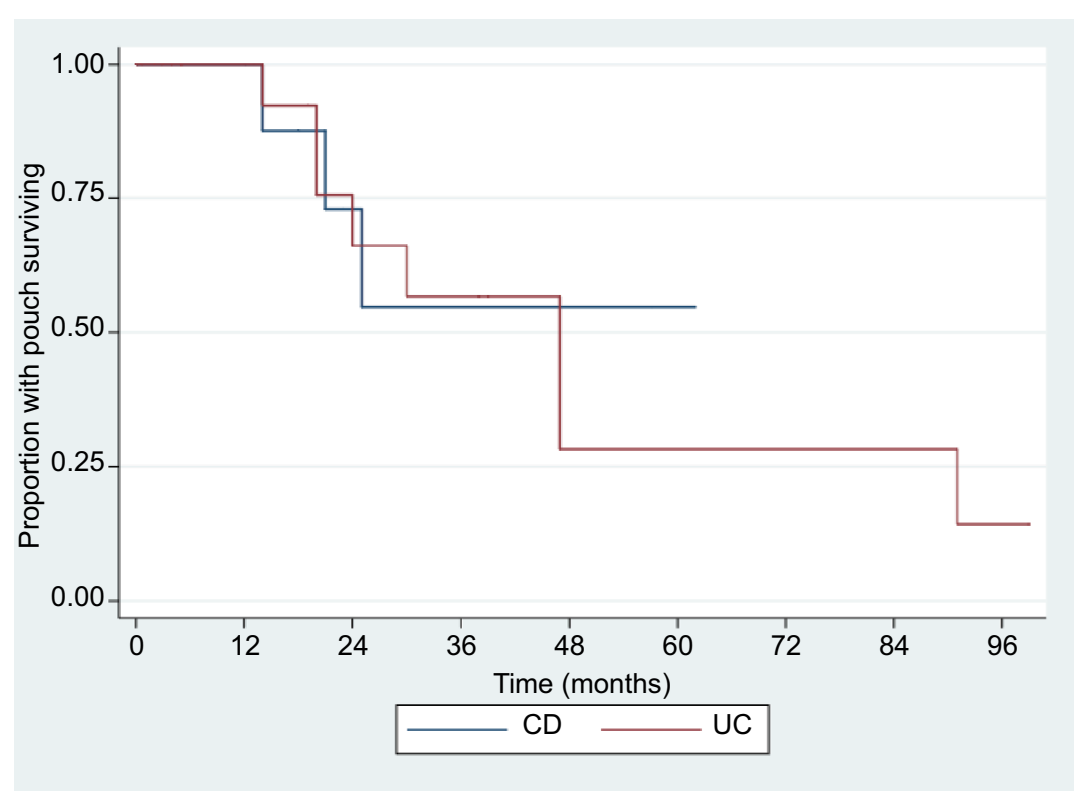

Figure I Kaplan-Meier graph for pouch failure.

Notes: Log rank test comparing "survival" times in the two groups $P=0.72$, no evidence of a difference between groups.

Abbreviations: CD, Crohn's disease; UC, ulcerative colitis.

highlighted that remission could be achieved in 53\%. ${ }^{18}$ Only under a quarter of patients achieved remission from their PPI when using a biologic, suggesting that the presence of PPI is associated with a less favorable treatment response.

It has been shown that PPI is often associated with pouchitis. ${ }^{8,11}$ As such it is difficult to know what additional contribution to symptoms is made by PPI, or if PPI in isolation requires any different treatment from pouchitis, or indeed should be classified as CD, as it effectively represents a skip lesion. It has however been demonstrated that PPI is likely to be a poor prognostic sign and associated with higher rates of pouch failure ${ }^{19}$ and therefore treatment with the aim of achieving remission may be associated with better outcomes.

This study has highlighted that PPI has practical and clinical implications. It is therefore essential that this complication is recorded during endoscopic pouch assessment.

This study is limited by small numbers and retrospective analysis. It is also limited by relative heterogeneity in patient cohort. Ideally future studies should explore comparing treatments for PPI in direct head to head trials. It is also surprising that no patients had prior exposure to a biologic. It has been reported that in patients with $\mathrm{UC}$ who require a biologic, those previously exposed to a biologic had poorer outcomes than those who had never previously been exposed to a biologic medication ${ }^{20}$ and so future work may help understand if this trend also occurs in those with PPI. A standard definition of PPI would also aid our understanding and outcomes of this complication, which has been shown to have a poorer prognosis than pouchitis in isolation.

\section{Conclusion}

To our knowledge, this is the largest study to explore the efficacy of all biologics for the specific treatment of PPI with the longest follow-up data. Biologics fail to achieve endoscopic remission of PPI in the majority of patients. In a small proportion of patients, they may help to prevent deterioration in pouch dysfunctional symptoms. In a large proportion of patients with PPI, surgery with indefinite diversion may be required despite biologic use.

\section{Acknowledgments}

This paper was presented in abstract form at the British Society of Gastroenterology Conference in Liverpool, UK, 2018 with interim findings. The poster's abstract was entitled "Biological therapy for the treatment of pre-pouch ileitis: a retrospective experience from three centres" and was published in "Poster Abstracts" GUT which can be found at: http://dx.doi.org/10.1136/gutjnl-2018-BSGAbstracts.183 (category: inflammatory bowel disease).

\section{Author contributions}

JPS, MR, CV, and RKF collected the data. JPS analyzed the data and prepared the manuscript. JPS and PB performed statistical analysis. JPS, MR, CV, RKF, GHTW, SDM, ALH, 
SKC, and ODF have revised the manuscript critically and prepared the final version of the manuscript. All authors contributed to data analysis, drafting and revising the article, gave final approval of the version to be published, and agree to be accountable for all aspects of the work.

\section{Disclosure}

The authors report no conflicts of interest in this work.

\section{References}

1. Sandborn WJ. Pouchitis: definition, risk factors, frequency, natural history, classification, and public health perspective. Trends Inflamm Bowel Dis Ther 1996. 1997.

2. de Buck van Overstraeten A, Wolthuis AM, Vermeire S, et al. Long-term functional outcome after ileal pouch anal anastomosis in 191 patients with ulcerative colitis. J Crohns Colitis. 2014;8(10):1261-1266.

3. Tulchinsky H, Dotan I, Halpern Z, Klausner JM, Rabau M. A longitudinal study of quality of life and functional outcome of patients with ulcerative colitis after proctocolectomy with ileal pouch-anal anastomosis. Dis Colon Rectum. 2010;53(6):866-873.

4. Lepistö A, Luukkonen P, Järvinen HJ. Cumulative failure rate of ileal pouch-anal anastomosis and quality of life after failure. Dis Colon Rectum. 2002;45(10):1289-1294.

5. Pardi DS, Shen B. Endoscopy in the management of patients after ileal pouch surgery for ulcerative colitis. Endoscopy. 2008;40(6):529-533.

6. Shen B. Pouchitis: what every gastroenterologist needs to know. Clin Gastroenterol Hepatol. 2013;11(12):1538-1549.

7. Samaan MA, de Jong D, Sahami S, et al. Incidence and Severity of Prepouch Ileitis. Inflamm Bowel Dis. 2016;22(3):662-668.

8. Mclaughlin SD, Clark SK, Bell AJ, Tekkis PP, Ciclitira PJ, Nicholls RJ. Incidence and short-term implications of prepouch ileitis following restorative proctocolectomy with ileal pouch-anal anastomosis for ulcerative colitis. Dis Colon Rectum. 2009;52(5):879-883.
9. Bell AJ, Price AB, Forbes A, Ciclitira PJ, Groves C, Nicholls RJ. Prepouch ileitis: a disease of the ileum in ulcerative colitis after restorative proctocolectomy. Colorectal Dis. 2006;8(5):402-410.

10. Segal JP, Ding NS, Worley G, et al. Systematic review with meta-analysis: the management of chronic refractory pouchitis with an evidence-based treatment algorithm. Aliment Pharmacol Ther. 2017;45(5):581-592.

11. Segal JP, Mclaughlin SD, Faiz OD, Hart AL, Clark SK. Incidence and long-term implications of prepouch ileitis: an observational study. Dis Colon Rectum. 2018;61(4):472-475.

12. Rottoli M, Vallicelli C, Bigonzi E, et al. Prepouch ileitis after ileal pouch-anal anastomosis: patterns of presentation and risk factors for failure of treatment. J Crohns Colitis. 2018;12(3):273-279.

13. Colombel JF, Ricart E, Loftus EV, et al. Management of Crohn's disease of the ileoanal pouch with infliximab. Am J Gastroenterol. 2003;98(10): 2239-2244.

14. Shen B, Remzi FH, Lavery IC, et al. Administration of adalimumab in the treatment of Crohn's disease of the ileal pouch. Aliment Pharmacol Ther. 2009;29(5):519-526.

15. Ferrante M, D'Haens G, Dewit O, et al. Efficacy of infliximab in refractory pouchitis and Crohn's disease-related complications of the pouch: a Belgian case series. Inflamm Bowel Dis. 2010;16(2):243-249.

16. Lightner AL, Pemberton JH, Loftus EJ. Crohn's Disease of the Ileoanal Pouch. Inflamm Bowel Dis. 2016;22(6):1502-1508.

17. Sandborn WJ, Tremaine WJ, Batts KP, Pemberton JH, Phillips SF. Pouchitis after ileal pouch-anal anastomosis: a Pouchitis Disease Activity Index. Mayo Clin Proc. 1994;69(5):409-415.

18. Segal JP, Ding NS, Worley G, et al. Systematic review with meta-analysis: the management of chronic refractory pouchitis with an evidence-based treatment algorithm. Aliment Pharmacol Ther. 2017;45(5):581-592.

19. Segal JP, Mclaughlin SD, Faiz OD, Hart AL, Clark SK. Incidence and long-term implications of prepouch ileitis: an observational study. Dis Colon Rectum. 2018;61(4):472-475.

20. Reinisch W, Sandborn WJ, Hommes DW, et al. Adalimumab for induction of clinical remission in moderately to severely active ulcerative colitis: results of a randomised controlled trial. Gut. 2011;60(6): 780-787.

\section{Clinical and Experimental Gastroenterology}

\section{Publish your work in this journal}

Clinical and Experimental Gastroenterology is an international, peerreviewed, open access, online journal publishing original research, reports, editorials, reviews and commentaries on all aspects of gastroenterology in the clinic and laboratory. This journal is included on PubMed. The manuscript management system is completely online

\section{Dovepress}

and includes a very quick and fair peer-review system, which is all easy to use. Visit http://www.dovepress.com/testimonials.php to read real quotes from published authors. 